\title{
An unobtrusive measurement method of the horizontal gaze angle
}

\author{
ANDREAS MÜHLBERGER \\ University of Tübingen, Tübingen, Germany \\ and \\ JÜRGEN A. REIß \\ Institut für Begleitforschung, Würzburg, Germany
}

\begin{abstract}
A horizontal gaze angle measurement device is introduced. By combining a photoelectric viewing device to measure the horizontal eye angle with a similar head angle measurement device, it is possible to measure the horizontal gaze angle without using a headrest. After discussion of circuit diagrams and measurement principles, it is shown that the measurements made with the device yield a reasonable precision. The mean absolute measurement error is below $1^{\circ}$. This inexpensive and unobtrusive device covers a visual field of about $20^{\circ}$ and can be used in parallel with many tasks. Further, data on the successful application of the device in a driving simulation setting are discussed.
\end{abstract}

Oculographic recording techniques provide a quantitative analysis and evaluation of eye movements in scientific studies as well as in routine eye movement assessmentsfor example, in clinical diagnosis. Shaunak, O'Sullivan, and Kennard (1995) listed the requirements of an ideal eye movement recording system as follows:

(a) Easy, nontraumatic application, ideally with no contact with the eye. (b) No interference with normal vision, and a sufficiently large field of vision. (c) Simultaneous measurement of horizontal, vertical and torsional eye movements. (d) High accuracy and repeatability, with a wide linear range of over $90^{\circ}$ of eye position. (e) High resolution allowing detection of eye movements as small as a few seconds of arc. (f) Good stability with no baseline drift. (g) Good dynamic measuring range (frequency bandwidth) of zero to a few hundred Hertz. (h) Insensitivity to translational head movements, and thus no need for rigid head fixation. (i) Insensitivity to surrounding levels of illumination, and to artifacts arising from blinks and electromyographic or electromechanical interference. (p. 121)

Additionally, we assume that cost of the device is an important variable. None of the currently available techniques fulfills all of these criteria (Shaunak et al., 1995). Thus, "the experimenter must compromise by choosing the method that is least inappropriate for the kind of investigation he or she wishes to make" (Carpenter, 1988, p. 405).

Correspondence should be addressed A. Mühlberger, Institute of Medical Psychology and Behavioral Neurobiology, University of Tübingen, Gartenstraße 2972074 Tübingen, Germany (e-mail: andreas. muehlberger@med.uni-tuebingen.de).

-Accepted by previous editor, Robert W. Proctor

\section{METHODOLOGIES FOR MEASURING EYE MOVEMENTS}

Five basic methodologies to measure eye movements are currently available. These are electrooculography, electromagnetic recording, photoelectric viewing, high-speed video recording, and devices using reflection.

\section{Electrooculography}

A usual covariate of eye movements is a changing electrical field in the tissues around the eyes. This field can be measured easily by applying electrodes at the upper and lower lids or left and right of the eye (at the external canthi). Advantages of electrooculography are the large measurement range of up to $\left( \pm 60^{\circ}\right.$ horizontally, and linearity for $\left( \pm 30^{\circ}\right.$. Furthermore, there are no limitations in the viewing field. Subjects can wear their glasses, and recordings can be made with closed eyelids (Shaunak et al., 1995).

Unfortunately, the recorded electric field is influenced not only by the movement of the eyes, but also by metabolic state and visual stimulation. Further, eye movements have been shown without corresponding changes in potential, and vice versa (Byford, 1963). The variability of the corneoretinal potential results in drifting of the baseline, particularly because of ambient illumination. Therefore, it is necessary to dark adapt the subject for $15-20 \mathrm{~min}$ before testing. The average sensitivity is only $1^{\circ}-2^{\circ}$ (Shaunak et al., 1995).

\section{Electromagnetic Recording}

Two (or more) pairs of coils are used, one attached to the eye and the other to the head. Each movement of the 
eye induces a current in the second coil; thus, the electromagnetic recording measures eye movement rather than eye position. The measurement is of high accuracy and can be made for all three axes. This measure, probably the most accurate, has a high sensitivity - a few seconds of arc. Its linear range is $\pm 20^{\circ}$ (Shaunak et al., 1995). Nevertheless, the accuracy of coil recordings in vivo is unknown (Merfeld, Black, \& Wade, 1998).

There are, however, disadvantages. First, the subject has to wear a scleral contact lens. Local anesthetic must be used for insertion, and insertion as well as removal may be unpleasant. Not all subjects or situations - for example, studies conducted in earth orbit-allow the use of this intrusive device (Merfeld et al., 1998). Furthermore, the coil can be left in place for only 20-30 min (DiScenna, Das, Zivotofsky, Seidman, \& Leigh, 1995). Second, the system as well as the coils are expensive (Shaunak et al., 1995). Third, subjects must remain in the center of the magnetic field. Therefore, research involving measurements during natural activities is impossible (DiScenna et al., 1995).

\section{Photoelectric Viewing}

A light-sensitive diode is adjusted to look at the highcontrast edge between the sclera (white) and the iris (dark). Concurrently, a small bulb or light emitting diode (LED) projects light at that very spot. Thus, the amount of light received by the sensor is more or less proportional to the amount of sclera illuminated by the source of light. Even in the crudest form, this device behaves linearly within a range of $10^{\circ}$ (Blakemore \& Carpenter, 1970).

The drawback of this method is that only the horizontal axis can be measured with satisfactory accuracy. Another problem is that photoelectric systems commonly work with the head fixed to get an accurate representation of direction of gaze (Shaunak et al., 1995). The potential sensitivity of the system is in the range of $3 \mathrm{~min}$ of arc, but in practice it is not quite so impressive (Shaunak et al., 1995).

\section{High-Speed Video Recorders}

A head-mounted video system is focused on the eye, mostly via an optical system to avoid restriction to the field of view. Software algorithms are used to locate the pupil center for each frame. Such devices yield a very high spatial resolution, but temporal resolution is still inadequate for full analyses, especially for microsaccades (Shaunak et al., 1995). The sampling rate is limited to $60 \mathrm{~Hz}$ with standard video equipment (in the United States; Merfeld et al., 1998). But technical development will solve this problem. Thus, a system with a sampling rate of $120 \mathrm{~Hz}$ $(180 \mathrm{~Hz}$ with head fixed) was described by DiScenna et al. (1995). A second disadvantage is that currently available systems are very expensive.

\section{Devices Using Reflection}

An external light source projects an image on the cornea of the eye that lies approximately $3.5 \mathrm{~mm}$ behind the corneal surface (the first Purkinje image). Any movement of the eye results in an apparent movement of this image, because the center of rotation of the eye is not identical to the center of the curvature of the cornea. Thus, recording the image of the light source yields information about the eye position.

A second technique using reflection is based on a reflection from attachments to the eye, usually a small mirror fitted on the eye with a contact lens. Both techniques allow measurement of the eye position in all three axes. Reflection devices are linear vertically and horizontally in $\pm 15^{\circ}$; they have a high temporal resolution, $300 \mathrm{~Hz}$, and an accuracy of a few minutes (Young \& Sheena, 1975).

\section{Comparison}

For a more detailed description of these measurement techniques, especially refinements and certain drawbacks, see Carpenter (1988). Müller, Cavegn, d'Ydewalle, and Groner (1993) compared electrooculography (EOG), photoelectric viewing, and two reflection devices. They concluded that all methods (except EOG) show reasonable temporal stability, with a mean absolute difference in degree of visual angle of approximately $1^{\circ}$ for a reflection device using a headrest and $1.4^{\circ}$ for photoelectric viewing. These methods have been applied in a wide variety of fields, including visual information processing, eye movement research, motion perception, and applied psychology (e.g., eye movements in pathology, sport, or traffic psychology).

Unfortunately, most of these methods do not involve a measure of head position. Such a measure is necessary for measuring natural gaze behavior, because naturally both head and eye movements are involved in gaze behavior. The common use of a bite bar or a headrest (Keith \& Sereno, 1994) results in artificial gaze behavior.

Yamada (1994) combined "an eye movement detection unit, a head movement detection unit, and a data processing unit" (p. 424) to solve this problem. Regrettably, he did not specify the exact nature or principles of the mentioned units. However, especially in traffic psychology, an unobtrusive apparatus is needed that allows the subject to move both the eyes and the head freely (Lovsund, Hedin, \& Tornros, 1991; Rahimi, Briggs, \& Thom, 1990). Further, measurement should be possible for several hours without substantial interference with the main task (e.g., driving).

\section{METHODOLOGIES FOR MEASURING HEAD MOVEMENTS}

Head orientation and position are the second substantial component in gaze behavior. Measurement devices for head position have been developed mainly in virtual reality research. Two different methodologies to measure head movements are currently available, measurement of head rotation and measurement of head translation.

\section{Measurement of Head Rotation}

Passive attitude detection systems are based on magnetic compass technology. They use the earth's gravity 


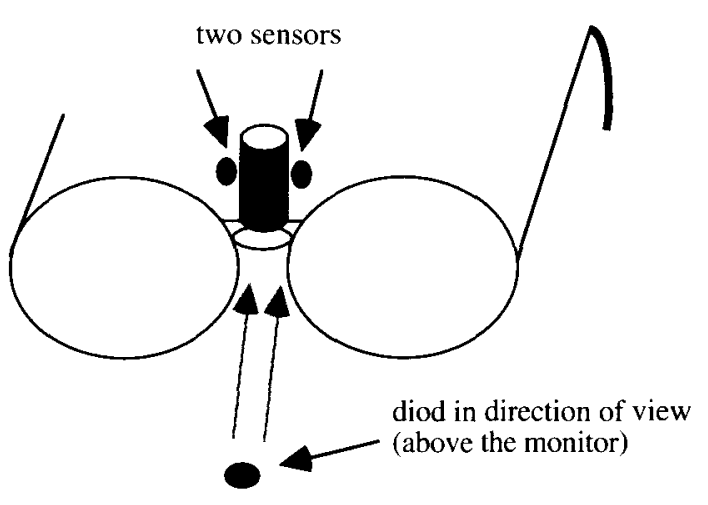

Figure 1. Measurement principle of the head angle. A diode $1 \mathrm{~m}$ in front of the subject emits a pulsed infrared signal. Two light-sensitive diodes mounted on the glasses receive this signal. The infrared light is partially shadowed by a black cylinder between the light-sensitive diodes. Any movement of the head around the vertical axis changes the signal received by the diodes. The relationship between the head angle and the difference between the received light on the left and right diodes is highly linear for about $20^{\circ}$.

and magnetic fields as references. No transmitters are required. For example, the CyperTrack Pro with $3 d f$ (degrees of freedom), by General Reality Company, yields an accuracy of $\pm 1.25^{\circ}$, a resolution of $0.15^{\circ}$, and a repeatability of $\pm 0.25^{\circ}$ for head heading. An accuracy of $\pm .25^{\circ}$, a resolution of $0.12^{\circ}$, and a repeatability of $\pm 0.25^{\circ}$ are realized for head tilt. The sampling rate is $30 \mathrm{~Hz}$ (Anonymous, 1999b).

The Wayfinder-VR (1995), also with $3 d f$, by Precision Navigation Inc., has an accuracy of $\pm 2^{\circ}$, a resolution of $0.1^{\circ}$, and a repeatability of $\pm 1^{\circ}$ for head heading. For head tilt, an accuracy of $1^{\circ}$, a resolution of $0.1^{\circ}$, and a repeatability of $\pm 0.5^{\circ}$ are realized. The sampling rate is $30 \mathrm{~Hz}$

\section{Measurement of Head Translation}

Retroreflective targets, illuminated by pulsed infrared light, are placed on the head. The reflected light appears as bright light spots. A high-performance computer calculates the head position on the basis of the size and position of these light spots. This can be performed with high sampling rates and high accuracy. For example, the ProReflex unit, available from Qualisys Products (1999), yields a sampling rate of up to $1000 \mathrm{~Hz}$. The DynaSight Sensor (1999) provides a sampling rate of $65 \mathrm{~Hz}$, a measurement resolution of $0.1 \mathrm{~mm}$ cross range, $0.4 \mathrm{~mm}$ down range, and a measurement accuracy of $2 \mathrm{~mm}$ cross range and $8 \mathrm{~mm}$ down range. These data are calculated with a $7-\mathrm{mm}$ target at $80-\mathrm{cm}$ range with a constant environment illumination.

\section{MEASURING HORIZONTAL GAZE ANGLE}

The basic idea behind the present method was to combine an eye movement device with an apparatus to mea- sure head movement. We were primarily interested in the horizontal gaze angle, because the method should be usable in a driving simulation scenario. Thus, for the eye measurement device we selected an improved infrared photoelectric viewing device, although Abel, Wall, Troost, and Black (1980) showed minor impairments in peripheral vision imposed by a photoelectric viewing device mounted on a spectacle. For the measurement of the horizontal head angle a new, inexpensive apparatus was developed. The core technique of this device is similar to the photoelectric viewing device.

\section{APPARATUS ${ }^{1}$}

\section{Measurement of the Horizontal Head Angle}

Measurement principle. An infrared LED is positioned $1 \mathrm{~m}$ in front of the subject. To measure the head position, two infrared light-sensitive diodes are mounted on a spectacle. The sensors are partly covered by a black cylinder; consequently, the infrared light reaches them only partly. If the head is turned to the right, the right diode is partially in the shade of the cylinder, while the left diode is exposed to the infrared light. After appropriate electrical amplification and filtering, the difference between the received luminous intensities of the right and left diodes is related linearly to the horizontal head angle. See Figure 1 for details.

Circuit diagram. Figure 2 shows the circuit diagram of the head angle measurement device. The device is a feedback control system, with the sum of light received by both diodes as the homeostatic variable. As an advantage, the difference in light intensity between the two diodes does not depend on the distance between the infrared LED and the light-sensitive diodes. To attain this, an output unit, input unit, and control unit are used:

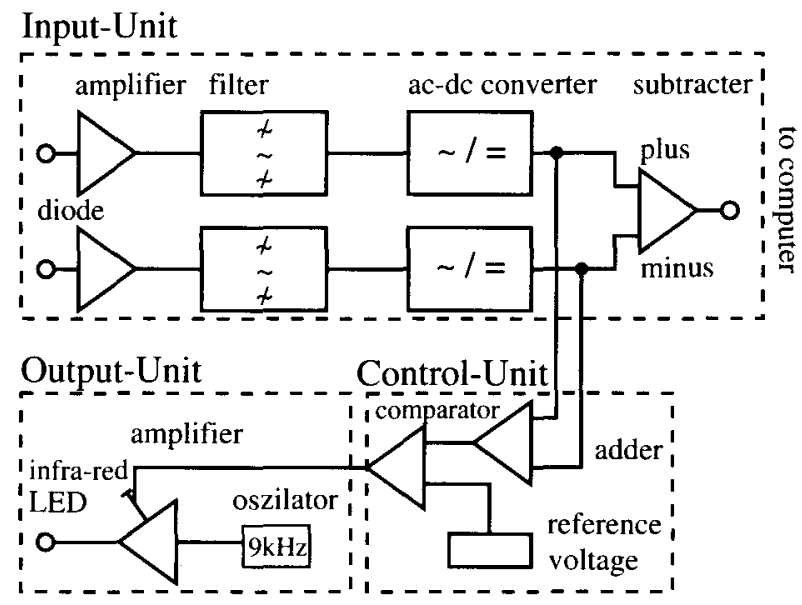

Figure 2. Circuit diagram of the head angle measurement device. The input unit consists of two light-sensitive diodes, two amplifiers, two filters, two ac-de converters, and one subtracter. The output unit (below) compares the input with a reference voltage and uses the difference to regulate the light-emitting diode (LED) driver amplifier of the output unit. 


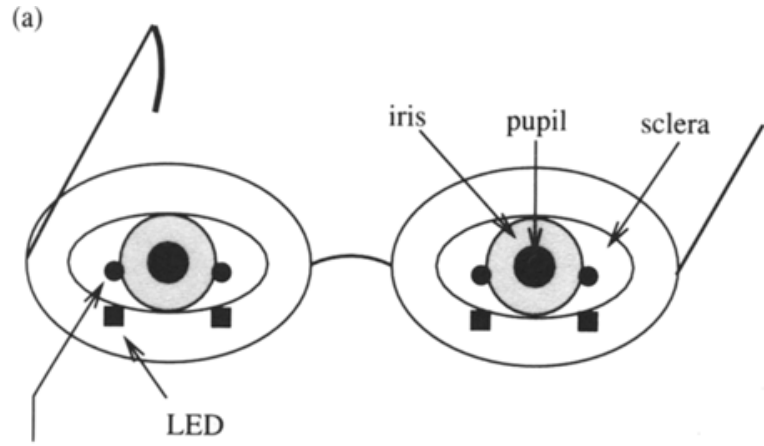

light-sensitive diode

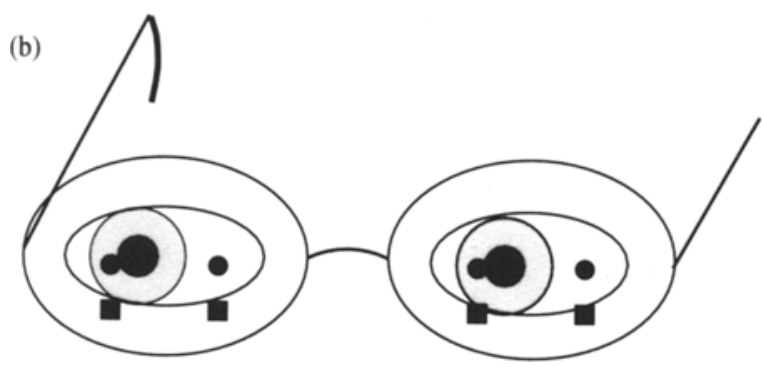

Figure 3. Measurement of the horizontal gaze angle. Infrared light emitting diodes (LED) and similar light-sensitive diodes (sensors) are adjusted to the left and right contrast edges between the sclera and iris for each eye. The voltage difference between the two sensors of the same eye is a linear function of the eye's gaze angle. The averaged input of both eyes increases the accuracy of the measurement. (a) the straight-ahead gaze; (b) a gaze to the right.

1. Output unit: An oscillator generates a $9-\mathrm{kHz}$ signal. The signal is amplified and drives an infrared LED with a maximal performance of $20 \mathrm{~mW}$.

2. Input unit: The light received by the light-sensitive diodes induces a voltage that is amplified, filtered, and ac-dc converted. The filtering of the signal is crucial in the whole process. Because the output unit generates a 9$\mathrm{kHz} \mathrm{AC}$ signal, the bandpass filter extracts the signal only. As a result, visual "noise" of the environment of the light-sensitive diodes cannot pass through this filter. The difference between the left and right diodes is the difference between the received luminous intensity of the right and left diodes. It is linearly related to the head angle for about $20^{\circ}$ and represents the output variable of the system.

3. Control unit: If no feedback loop between input and output were provided, any movement of the subject to or away from the LED would result in a change in the difference between the left and right light-sensitive diodes. Thus, the sum of the left and right output has to be as constant as possible. Therefore it is compared with a reference voltage. The difference between reference voltage and output voltage serves as an input for the amplifier of the infrared LED. This rather simple feedback control loop is sufficient because the distance between the LED and the light-sensitive diodes is more than 10 times bigger than the expected movement of the subjects' head in the longitudinal direction.

\section{Measurement of the Horizontal Eye Angle}

The measurement device of the eye positions (see Figure 3 ) is an implementation of the photoelectric viewing device just described. To improve the quality of the measurement the following improvements have been made:

1. The LEDs do not emit a constant light; rather, it is pulsed with $4 \mathrm{kHz}$ to isolate the light due to the LED from the light due to the room illumination.

2. Similar to the head angle measurement device, a feeback control loop adjusts for any differences in distance of the LED from the eye during the experiment.

3. A small black tube around both sensors and LEDs excludes light not directly reflected from the eyes.

The circuit diagram of the measurement device for the horizontal eye position is essentially the same as for horizontal head angle. The difference between the received luminous intensity of the right and left diodes is linearly related to the horizontal eye angle for about $12^{\circ}$.

\section{EXPERIMENT}

The goals of the present experiment were twofold: (1) to obtain an estimation of the validity and accuracy of the measurement device; and (2) to apply the measurement device as a new method in an experimental driving simulation setting.

\section{Method}

Subjects and Design. Six subjects participated in three 1-h sessions each. Participation in the experiment was part of a course requirement, although subjects were free to choose one of several experiments. Two of the subjects were male, and 4 were female. The average age was $28.8(S D=3.62)$ years. The first session functioned as a training session to learn to drive with the driving simulation. With respect to the two main goals, the experiment consisted

Table 1

Overview of the Design

Subjects 1-3

Subjects 4-6

Session 1 introduction to driving task introduction to driving task driving in silence driving while talking

Session 2 line-of-sight task driving in silence line-of-sight task driving while talking line-of-sight task

Session 3 line-of-sight task driving while talking line-of-sight task driving in silence line-of-sight task driving in silence driving while talking line-of-sight task driving while talking line-of-sight task driving in silence line-of-sight task line-of-sight task driving in silence line-of-sight task driving while talking line-of-sight task

Note-During Session 1, subjects were introduced to the driving task. In the following sessions, the driving task was embedded in line-ofsight task blocks. Each block consisted of four lines of sight task trials. 


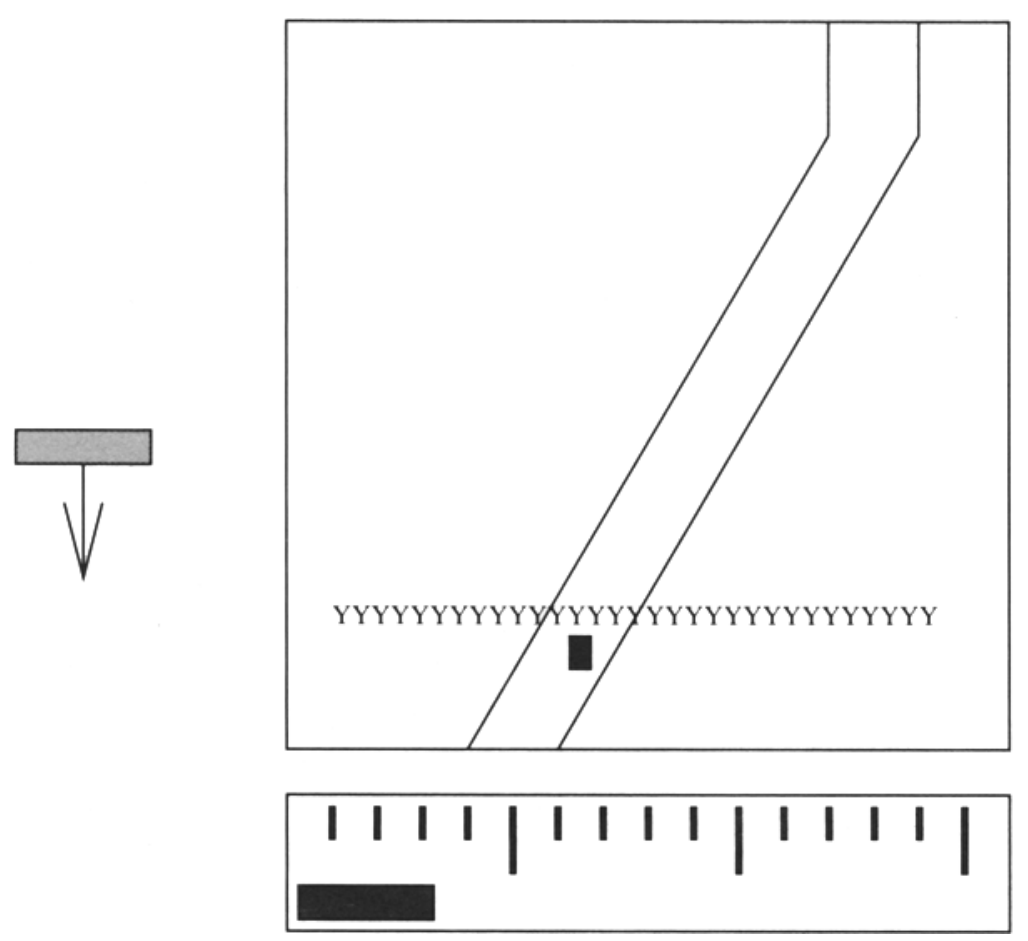

Figure 4. The driving simulation task. Representation of the screen while a peripherally warned barrier appears. The subject can anticipate these barriers that appear straight before the car while the peripheral bars are at the same height.

of two tasks, a line-of-sight task interspersed with a driving simulation task (Table 1).

Line-of-sight task. During the line-of-sight task, the subject was seated in a car seat approximately $1 \mathrm{~m}$ away from a computer screen. The task was to fixate a 4-mm large red point moving horizontally for $22.8 \mathrm{~cm}$ on the screen, thus resulting in an approximate $12^{\circ}$ movement in the visual field. The speed of the point was $1.2^{\circ}$ per second. There were four subtasks:

1. The point moves from left to right, and the subject should follow the point with the eyes, leaving the head in a position as constant as possible.

2. The point moves from right to left, and the subject should follow the point with the eyes, leaving the head in a position as constant as possible.

3. The point moves from left to right, and the subject should follow the point with the head, leaving the eyes straight.

4. The point moves from right to left, and the subject should follow the point with the head, leaving the eyes straight.

A trial consisted of one of each of the four subtasks. Four trials made up a block.

The subject was instructed not to blink. A subtask required about $12 \mathrm{sec}$, while the interval between subtasks was self-paced. The total duration of a line-of-sight task block was about $4 \mathrm{~min}$. Thus, for each subject, 24 measurements (two sessions, three blocks, and four trials) were available. The line-of-sight task was conducted at the beginning, in the middle, and at the end of Sessions 2 and 3.

The driving simulation task. The hardware of the driving simulation task is a car seat, steering wheel, gas and brake pedals, and a 486-50 personal computer with a 14 -in. screen. The subject views the road and car from a bird's-eye view. The software presents the street and the car in text mode $(80 \times 52)$; thus, the car is a blue square on a street of red $x$ characters. The street consists of two ver- tical downscrolling curved lines with 8 characters of space between (about $22 \mathrm{~mm}$ street width). This results in about $1.2^{\circ}$ of the subject's visual field. The distance of the peripheral bars to the road depends on the curve situation, but is constant with respect to the screen. The horizontal distance from the middle of the screen was about $80 \mathrm{~mm}$ or $4^{\circ}$ in the subject's visual field.

During driving, the subject encounters so-called barriers, which are $\mathrm{Y}$ characters arranged in a row in front of the car, that are displayed for exactly $1 \mathrm{sec}$. This should simulate an event that blocks the road. The task is to avoid driving into the barriers. If a subject hits a barrier, this event is scored as an accident. If the subject has an accident, he/she has to drive backward for $20 \mathrm{sec}$ at a speed of $60 \mathrm{~km} / \mathrm{h}$. This penalty was introduced to punish high-speed driving. Dependent measures are the accident risks and the speed.

Two kinds of barriers may appear: unwarned barriers and peripherally warned barriers. Unwarned barriers emerge $50 \mathrm{~m}$ in front of the car. In order to avoid an accident with unwarned barriers, the subject is instructed to drive $80 \mathrm{~km} / \mathrm{h}$ or slower. Depending on speed, the subject has to either brake or do nothing (the barrier vanishes after $1 \mathrm{sec}$ ). Peripherally warned barriers are announced by a bar at the left and right margins of the screen. The bars are moving up and down independently, and every time the bars are at exactly the same height, a barrier arises $5 \mathrm{~m}$ in front of the car (Figure 4). The subject is instructed that whenever the bars come close to each other, he/she has to bring the car to a full stop to let the barrier pass. Thus, to avoid accidents the subject has to look occasionally toward the peripheral parts of the screen (for a more detailed description of the driving simulation task, see Reiß, 1997; Reiß \& Krüger, 1995).

The basic question for this experiment was whether an accident during a peripherally warned barrier happens because the subject (1) does not see the warning (in this case the subject does not see that the bars at the left and right of the screen are at the same height) or (2) does see the warning but does not react properly. If the subject does not look at the warning - that is, does not direct her/his 
Table 2

Results of the Multiple Regressions From the Voltage Data on the Gaze Angle

\begin{tabular}{|c|c|c|c|c|c|c|c|c|}
\hline & \multicolumn{2}{|c|}{$r^{2}$} & \multicolumn{2}{|c|}{ Intercept $b_{0}$} & \multicolumn{2}{|c|}{ Eye $b_{1}$} & \multicolumn{2}{|c|}{ Head $b_{2}$} \\
\hline & $M$ & $S D$ & $M$ & $S D$ & $M$ & $S D$ & $M$ & $S D$ \\
\hline \multicolumn{9}{|c|}{ Session } \\
\hline 1 & .973 & .026 & -54.7 & 25.9 & .038 & .016 & -.0063 & .0018 \\
\hline 2 & .972 & .025 & -60.1 & 30.5 & .041 & .014 & -.0054 & .0013 \\
\hline \multicolumn{9}{|c|}{ Block } \\
\hline 1 & .977 & .021 & -52.4 & 26.7 & .037 & .015 & -.0058 & .0016 \\
\hline 2 & .973 & .028 & -51.9 & 26.8 & .037 & .014 & -.0059 & .0016 \\
\hline 3 & .967 & .026 & -67.8 & 29.1 & .044 & .015 & -.0059 & .0017 \\
\hline \multicolumn{9}{|c|}{ Trial } \\
\hline 1 & .978 & .018 & -56.1 & 26.9 & .038 & .014 & -.0059 & .0016 \\
\hline 2 & .971 & .027 & -58.0 & 28.9 & .040 & .016 & -.0058 & .0016 \\
\hline 3 & .971 & .028 & -57.2 & 29.1 & .040 & .015 & -.0058 & .0017 \\
\hline 4 & .970 & .027 & -58.3 & 29.6 & .039 & .015 & -.0058 & .0017 \\
\hline \multicolumn{9}{|c|}{ Subject } \\
\hline 1 & .986 & .011 & -25.1 & 5.3 & .023 & .002 & -.0038 & .0002 \\
\hline 2 & .986 & .016 & -79.7 & 17.3 & .047 & .009 & -.0046 & .0003 \\
\hline 3 & .940 & .023 & -64.6 & 20.1 & .043 & .008 & -.0070 & .0026 \\
\hline 4 & .963 & .024 & -41.4 & 14.2 & .032 & .008 & -.0064 & .0005 \\
\hline 5 & .985 & .007 & -89.2 & 23.6 & .061 & .012 & -.0068 & .0004 \\
\hline 6 & .974 & .027 & -44.3 & 18.3 & .031 & .009 & -.0065 & .0003 \\
\hline$M$ & .972 & .025 & -57.4 & 28.3 & .039 & .015 & -.0058 & .0016 \\
\hline
\end{tabular}

Note-The mean and standard deviation of the explained variance $r^{2}$, intercept $b_{0}$, and the coefficients $b_{1}$ and $b_{2}$ for the eye voltage $\varepsilon$ and the head voltage $\tau$ are reported for the sessions, blocks, trials, and subjects. The total sample size was 144 measurements. Thus, 24 measurements were available for each subject.

gaze in an angle between $3^{\circ}$ and $6^{\circ}$ - the subject should not see the warning either. Our hypothesis was that the probability for a gaze in an angle between $3^{\circ}$ and $6^{\circ}$ would be small during a peripheral warning in case of an accident and high if an accident is avoided. No such difference should be found for unwarned barriers.

Subjects had to drive twice for $22 \mathrm{~min}$ in Sessions 2 and 3. The experimenter was present on both occasions. In one trip he was just a silent observer of the driving behavior, whereas in the other trip the subject and the experimenter were instructed to talk to each other. The results of the manipulated speech behavior are reported elsewhere (Reiß, 1997).

\section{Results}

The line-of-sight task. Because the subject is asked to look at a well-defined spot on the screen (target), we assume that the subject looks at the target. Thus, for simplification, the term target angle is used to refer to the angle of the point on the screen, and the term gaze angle is used to refer to the predicted gaze angle. Thus, the horizontal gaze angle could be predicted with the three voltages from the two eye angles and the head angle. The regression from the voltages on the target angle is interpreted as an external validity measure.

To demonstrate the usefulness of the method, the regression equation of the first trial was used to estimate the gaze angle on subsequent trials. The correlation between these predicted gaze angles and the actual target angle is a measure of the stability of the method.
The sampling rate for the line-of-sight task was $20 \mathrm{~Hz}$ for a total duration of $12.9 \mathrm{sec}$ across four different subtasks, resulting in approximately 1,032 data points for a trial. Any invalid data, mainly eye blinks, were excluded from the analysis. As a result at least 739 , and in most cases more than 900 , measurements remained for each trial. Note that one block of the line-of-sight tasks consists of four trials, with three blocks being administered during one session.

Multiple regressions between voltage input and the angle of the spot to be looked at. For each line-of-sight task a multiple regression between the voltage input and the target angle was computed with SAS (Statistical Analysis System). The predictors were the voltages measured on both eyes $(\varepsilon)$ and head $(\tau)$. The predicted value $(\theta)$ is the target angle (in degrees). The target angles were between $-6^{\circ}$ and $+6^{\circ}$. The following equation was used: ${ }^{2}$

$$
\theta=b_{0}+b_{1} \varepsilon+b_{2} \tau \text {. }
$$

The goal of the multiple regression was to obtain the parameters $b_{0}, b_{1}$, and $b_{2}$ of the regression. Table 2 shows the results of the multiple regression. The averaged explained variance, $r^{2}$, is always at least .94 and in most cases higher than .97. Further, the stability of all parameters was rather high across trials, whereas between sessions and blocks, considerable differences occurred (e.g., a shift in the head angle gradient of nearly $0.5 S D$ occurred from Session 2 to 3). As usual with physiological data, the parameters differed greatly between subjects, whereas the variability within subjects in terms of standard deviations was smaller than the variability between subjects. Thus, it is necessary to adjust the measurement device to the subject and perform a baseline measurement each session.

Cross-validities. As noted, we computed the parameters estimated in the first trial of any line-of-sight task block and used them to predict the gaze angle in subsequent trials. Thus, we computed $b_{0}, b_{1}$, and $b_{2}$ for each first trial of each block and subject, and estimated the gaze angle $\hat{\theta}$ with Equation 1 and the voltage data $\varepsilon$ and $\tau$ of the eye and head in subsequent trials.

The estimated gaze angles $\theta$ (using the parameters of a previous trial) were correlated with the actual target angle $\theta$ :

$$
\theta=a_{0}+a_{1} * \hat{\theta}
$$

If the prediction were perfect, the resulting intercept $a_{0}$ would be zero and the gradient $a_{1}$ would be one. The intercept might be interpreted as the systematic bias of the prediction, while the gradient reflects variable errors.

Table 3 shows the results. The explained variance is very high (mostly higher than .95) for each of the 18 equations per subject, but this only describes relative quality. The crucial variables are the regression parameters. The average deviation of the intercept $a_{0}$ from zero is only 
Table 3

Regression Parameters Between Predicted Gaze Angle Using Regression Parameters of the First Trial in a Block and Actual Gaze Angle

\begin{tabular}{|c|c|c|c|c|c|c|}
\hline & \multicolumn{2}{|c|}{$r^{2}$} & \multicolumn{2}{|c|}{ Intercept $a_{0}$} & \multicolumn{2}{|c|}{ Gradient $a_{1}$} \\
\hline & $M$ & $S D$ & $M$ & $S D$ & $M$ & $S D$ \\
\hline \multicolumn{7}{|l|}{ Session } \\
\hline 2 & .978 & .020 & -.0717 & .2350 & .9633 & .0532 \\
\hline 3 & .954 & .135 & .0737 & .2780 & .9606 & .0588 \\
\hline \multicolumn{7}{|l|}{ Block } \\
\hline 1 & .981 & .019 & .0145 & .2756 & .9614 & .0418 \\
\hline 2 & .946 & .164 & .0065 & .1710 & .9642 & .0622 \\
\hline 3 & .971 & .028 & -.0180 & .3335 & .9601 & .0625 \\
\hline \multicolumn{7}{|l|}{ Trial } \\
\hline 2 & .975 & .027 & .0119 & .2754 & .9629 & .0631 \\
\hline 3 & .948 & .164 & -.0187 & .2678 & .9622 & .0502 \\
\hline 4 & .974 & .024 & .0099 & .2624 & .9606 & .0549 \\
\hline \multicolumn{7}{|l|}{ Subject } \\
\hline 1 & .991 & .007 & .0288 & .1662 & .9692 & .0295 \\
\hline 2 & .929 & .234 & .1875 & .2851 & .9899 & .0288 \\
\hline 3 & .946 & .030 & .1213 & .2512 & .9504 & .0598 \\
\hline 4 & .967 & .017 & -.2531 & .2401 & .9434 & .0703 \\
\hline 5 & .985 & .010 & -.0486 & .1558 & .9773 & .0554 \\
\hline 6 & .978 & .021 & -.0297 & .2605 & .9413 & .0651 \\
\hline Average & .966 & .097 & .0010 & .2664 & .9619 & .0558 \\
\hline
\end{tabular}

Note-The mean and standard deviation of the explained variance $r^{2}$ intercept $a_{0}$, and gradient $a_{1}$ are given for sessions, block, trial, and subjects. The total sample size was 108 measurements. Thus, 18 measurements were available for each subject.

$0.001^{\circ}$, whereas the standard deviation indicates considerable variability. Assuming a normal distribution, a standard deviation of $0.266^{\circ}$ yields a bias range of approximately $-0.5^{\circ}$ to $+0.5^{\circ}$ for $95 \%$ of the measurements. Especially if predictions of the behavior for single subjects are required, it seems essential to perform a small baseline segment within the to-be-predicted task.

The mean value of the gradient $a_{1}(.9619)$ was quite satisfactory, too. For $95 \%$ of the measurements, a gradient higher than .85 is to be expected.

Confidence intervals. In any measurement of the horizontal gaze angle, a basic question concerns the average error for predicted raw scores. According to Hays (1988), the interval estimation in linear regression for raw scores of the measurement $j$ is given by ${ }^{3}$

$$
\begin{aligned}
& s_{y \cdot x}^{2}=s_{y}^{2}-b_{y \cdot x}^{2} s_{x}^{2}, \\
& \hat{s}_{y \cdot x}^{2}=\frac{N}{N-2} s_{y \cdot x}^{2},
\end{aligned}
$$

and

$$
C I=y_{j} \pm t_{(\alpha / 2)} \hat{s}_{y \cdot x}^{2} \sqrt{1+\frac{1}{N}+\frac{\left(x_{j}-m_{x}^{2}\right)}{N s_{x}^{2}}} .
$$

Where $b_{y . x}{ }^{2}$ is the squared regression coefficient, $y_{j}$ is the predicted value, $x_{j}$ is the predictor, and $N$ is the sample size.
In the present case the variances $s_{x}^{2}$ and $s_{y}^{2}$ were approximately $3.2^{2}=10.24$, the number of data points $N$ was at least 700 , and the maximal quadratic deviation $\left(x_{j}-m\right)^{2}$ was $(6-0)^{2}=36$. According to Table 3 , the average gradient was .96 , thus $b_{v \cdot x}^{2}=.92$. This results in

$$
\begin{aligned}
& s_{y . x}^{2}=10.24-.92 * 10.24=.82, \\
& \hat{s}_{y . x}^{2} \leq \frac{700}{698} * .82=.82,
\end{aligned}
$$

and

$$
\begin{aligned}
C I & =y_{j} \pm t_{0.975} .82 \sqrt{1+\frac{1}{700}+\frac{36}{700 * 10.24}} \\
& =y_{j} \pm 1.96 * .82 * 1.003=y_{j} \pm 1.61 .
\end{aligned}
$$

Thus, the predicted gaze angle is in $95 \%$ of the predictions of any single data point nearer than $1.61^{\circ}$ to the actual gaze angle. To illustrate the prediction graphically, the regression equation and its confidence limits for individual scores are shown in Figure 5.

Line of sight during the driving simulation task. During the driving simulation task, the subject was wearing the gaze angle measurement device. Because of the specific situation, the subject did not always look at the screen. Thus, any of the following events were used to indicate invalid data, which were excluded from the analysis: (1) The subject was not looking at the screen, (2) the subject's eyes were closed, (3) the subject was looking at the speedometer, or (4) the subject just had an accident and now had to drive backward. After this procedure, $228 \mathrm{~min}$ of the total 480 available minutes could be analyzed.

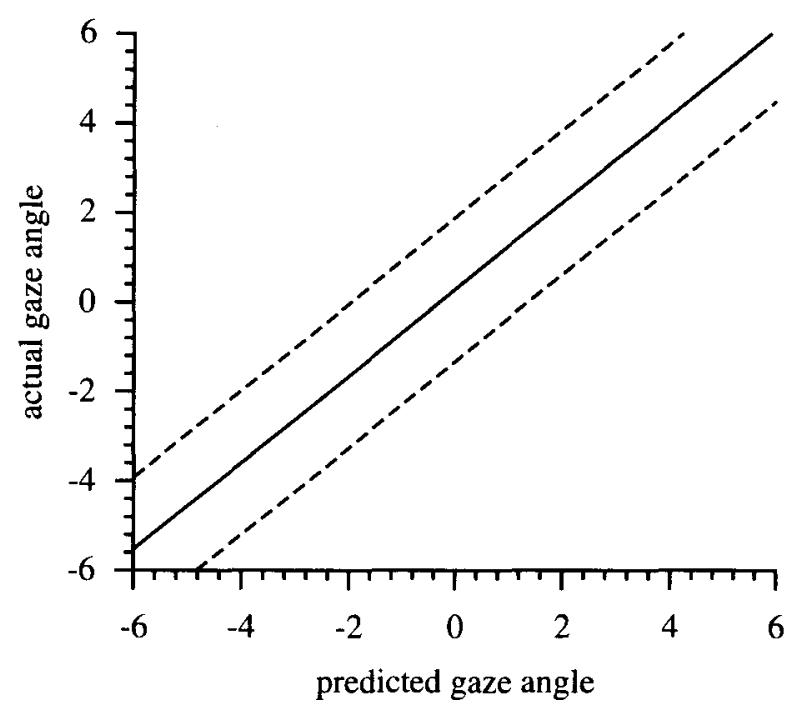

Figure 5. Regression between predicted and actual gaze angle. Regression between predicted and actual gaze angles for Subject 1 over all conditions. The dashed lines represent the $95 \%$ confidence intervals for individual raw scores. 


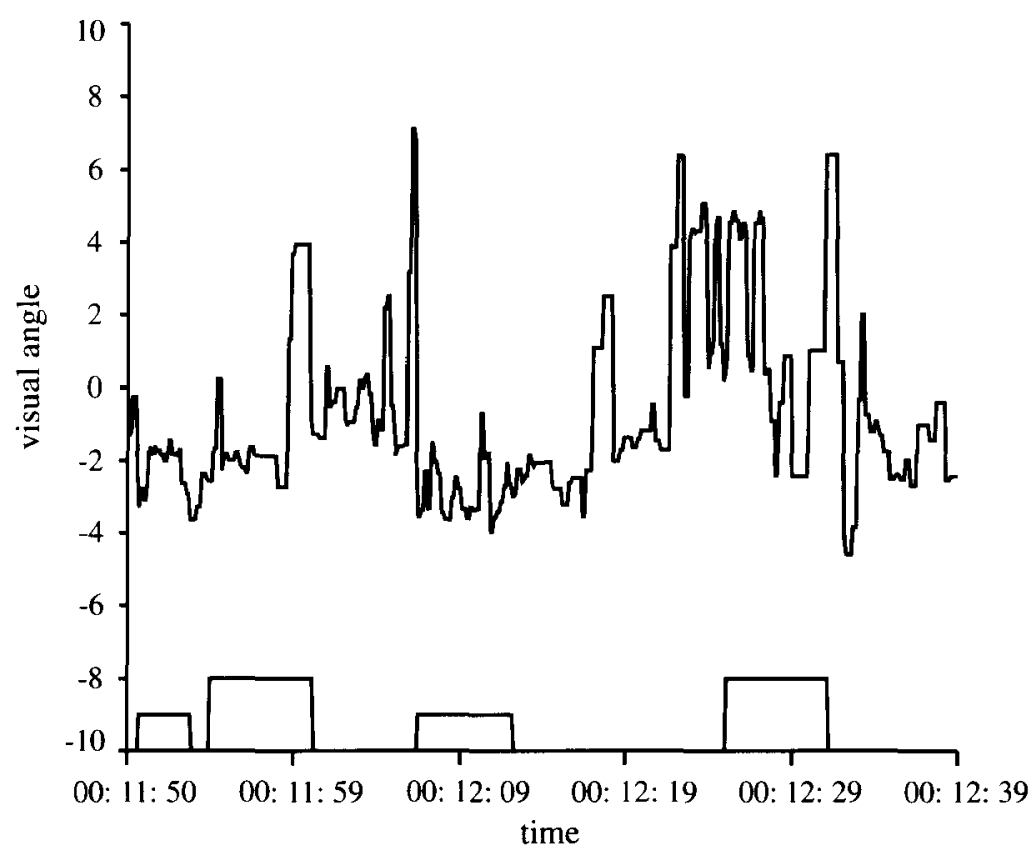

Figure 6. Gaze angle diagram of a subject during a short driving interval. The driving situation is indicated by the bottom line: $A$ value of -9 indicates a short episode without warning, while a value of -8 indicates a situation announcing a peripherally warned barrier. The pattern of the gaze angle suggests that inspections of the visual periphery are more probable before and during the warning.

To compute the gaze angle during a trip, the linear equation (1) of the immediately preceding line-of-sight task block was used. Further, the difference of the resulting angle and the angle of the position of the car relative to the subject was computed for each time sample. To compensate for mean constant errors (e.g., movement of glasses), the median of the resulting time series was computed and taken of each data point.

Figure 6 gives a brief sample of the visual inspection pattern applied. It shows that the subject relied heavily on the scanning of the periphery while driving in a peripherally warned situation. During the absence of any warning, scanning of the periphery is scarce. Peripheral scanning occurs only to assure the subject of the absence of any danger.

For each subject the probability of a gaze angle between $3^{\circ}$ and $6^{\circ}$ was computed for peripherally warned barriers and unwarned barriers if an accident occurred or did not occur, respectively. The probability was estimated by dividing the number of samples with a gaze angle in the critical region between $3^{\circ}$ and $6^{\circ}$ by the total number of samples. The probabilities are shown in Figure 7.

An analysis of variance showed a tendency for the gaze position to be less often directed in the periphery if an accident was about to happen $[F(1,5)=6.32, p=.054]$. Further, the interaction between accident and situation indicates that if the driver is capable of avoiding an accident, the scanning of the visual periphery is very probable $[F(1,5)=9.18, p<.05]$.

\section{DISCUSSION}

The results show clearly that the present device is able to measure the gaze angle in a mainly unrestricted setting. Even though no headrest was used, the confidence interval of the mean absolute difference (the intercept) had a width of less than $1.2^{\circ}$, which is well below the results of Müller et al. (1993). Even for a single measurement, the width of the $95 \%$ confidence interval is quite small. This high accuracy was obtained by using a feedback loop in both the head angle and the eye angle LEDs. The sampling rate of the device was only $20 \mathrm{~Hz}$ for this investigation. Thus, it is possible to enhance accuracy by increasing the sampling rate up to at least $200 \mathrm{~Hz}$.

Although the device does not meet all the criteria of an ideal measurement system for eye movements, it has some important advantages. First, the device combines measures of eye position and head orientation. Second, the system is inexpensive. Third, the system is very light (about $40 \mathrm{~g}$ ). Fourth, it is simple to adapt and allows a nearly unaffected field of view. Fifth, it is possible to use the system in natural situations outside the laboratory (e.g., driving).

The accuracy of the device is not as good as that of other devices ( $1.6^{\circ}$ with target distance of $1 \mathrm{~m}$ or more). However, it seems possible to use this low-cost device in a wide variety of experimental and applied visual and clinical psychophysics, and in clinical diagnostics. As a demonstration of an application, we used a driving simu- 


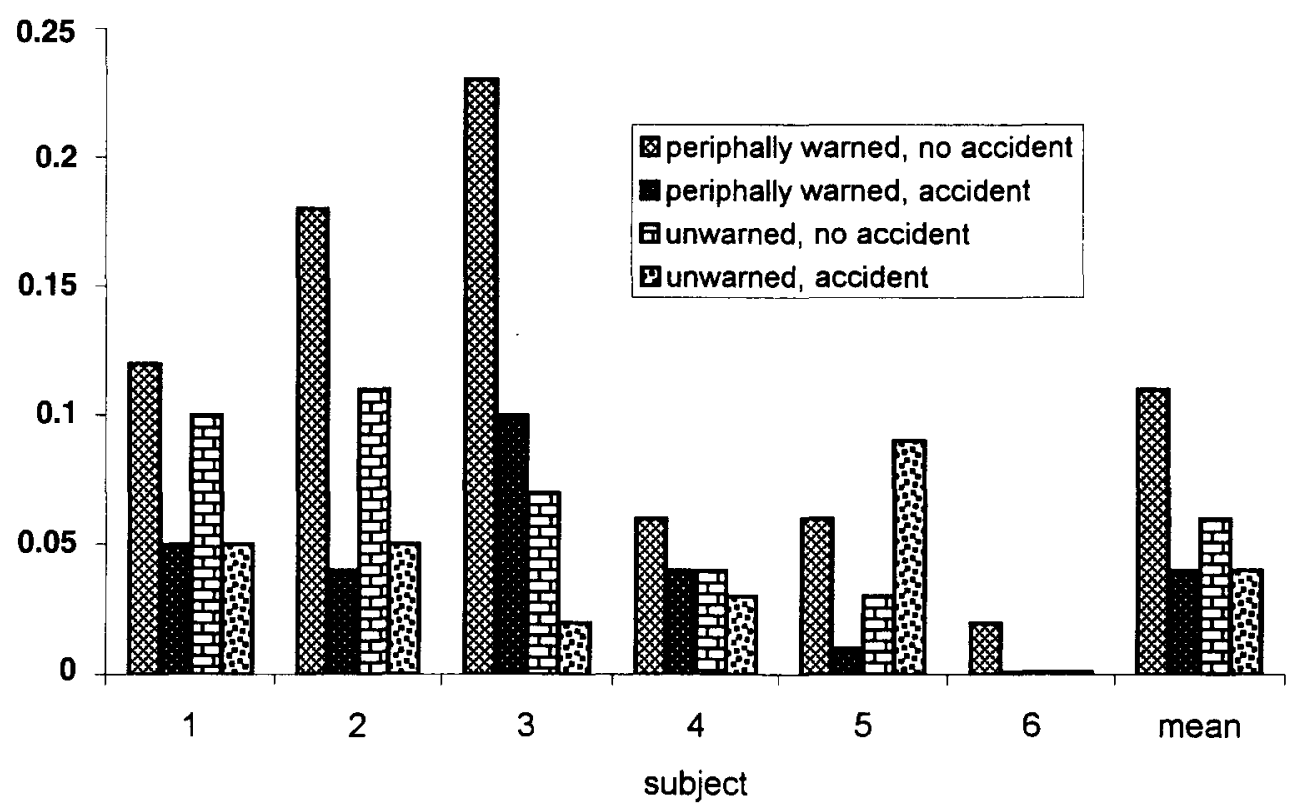

\begin{abstract}
Figure 7. Probability of a gaze angle between $3^{\circ}$ and $6^{\circ}$. Computed for peripherally warned barriers and unwarned barriers that led to an accident versus no accident. According to expectation, the probability is very high in the peripherally warned and no-accident conditions.
\end{abstract}

lation paradigm. In driving it is impossible to use a headrest, although the gaze angle is a crucial variable. Further, the measurement of the gaze angle must be applicable for $1 \mathrm{~h}$ or longer, which is possible only if the measurement device is unobtrusive. By combining photoelectric viewing with a similar head angle measurement device, we were able to meet these criteria. We showed that accidents in the very specific driving simulation situation described here were due to lacking information input from periphery rather than deficits in information processing. This is an important result to disentangle the cognitive processes necessary for avoiding accidents. Such results could help to improve road safety.

Several shortcomings of the device are not yet overcome: First, the horizontal gaze angle is measured in a range of $20^{\circ}$ only. Second, the device allows no measurement of the vertical gaze angle and head translations. So the device is usable in experiments with small head translations only (e.g., subject is seated) in relation to viewing distance. Third, there is still a considerable variability between blocks, indicating that the subjects shifted the position of the spectacles. Directions for further development are to (1) miniaturize and optimize the device (e.g., extend the measurement range), (2) integrate measurement of eyelid closure, and (3) develop or implement a device for measuring vertical head movements and head translations.

\section{REFERENCES}

Abel, L. A., Wall, III, C., Troost, B. T., \& Black, F. O. (1980). Dependence of optokinetic nystagmus characteristics upon recording techniques. Awation, Space \& Environmental Medicine, 51, 1112-1113.
Blakemore, C. B., \& Carpenter, R. H. S. (1970). A very simple device to measure human eye movements. Journal of Physiology, 209, $155-178$.

BYFORD, G. H. (1963). Non-linear relations between the corneo-retinal potential and horizontal eye movements. Journal of Physiology, 168, 14-15.

Carpenter, R. H. S. (1988). Movement of the eyes. London: Pion.

DiScenna, A. O., Das, V., Zivotofsky, A. Z., Seidman, S. H., \& LEIGH, R. J. (1995). Evaluation of a video tracking device for measurement of horizontal and vertical eye rotations during locomotion. Journal of Neuroscience Methods, 58, 89-94.

The DynaSight Sensor [On-line] (1999). Available: www.orin.com/ 3dtrack/dyst.htm

General Reality Company CyberTrack Product Descriptions [On-line] (1999). Retrieved 4/17/99 at: www.genreality.com/cybertrack sheet.html

Hays, W. L. (1988). Statistics. New York: Holt, Rinehart \& Winston. Keith, R., \& Sereno, S. C. (1994). Eye movements in reading: Psycholinguistic studies. In M. A. Gernsbacher (Ed.), Handbook of psycholinguistics (pp. 57-81). San Diego: Academic Press.

Lovsund, P., Hedin, A., \& TornRos, J. (1991). Effects on driving performance of visual field defects: A driving simulator study. Accident Analysis \& Prevention, 23, 331-342.

Merfeld, D. M., Black, F. O., \& WadE, S. (1998). Clinical use of threedimensional video measurements of eye movements. OtolaryngologyHead \& Neck Surgery, 118, S35-S38.

Müller, P. U., Cavegn, D., D'Ydewalle, G., \& Groner, R. (1993). A comparison of a new limbus tracker, corneal reflection technique, purkinje eye tracking and electro-oculography. In G. d'Ydewalle \& J. van Rensbergen (Eds.), Perception and cognition (pp. 393-401). Amsterdam: Elsevier.

Qualisys Products [On-line] (1999). Retrieved 4/17/99 at: www.qualisys. com/products.htm

Rahimi, M., Briggs, R. P., \& Thom, D. R. (1990). A field evaluation of driver eye and head movement strategies toward environmental targets and distractors. Applied Ergonomics, 21, 267-274.

REIß, J. A. (1997). Das Unfallrisiko mit Beifahrer: Der Beifahrer als Doppelaufgabe. In E. van der Meer, T. Bachmann, R. Beyer, C. Goertz, H. Hagendorf, B. Krause, W. Sommer, H. Wandke, \& M. Zießler 
(Eds.), Experimentelle psychologie (pp. 401-402). Lengrich, Germany: Papst Science Publishers.

Reib, J. A., \& KrüGER, H.-P. (1995). Accident risk modified by passengers. In C. N. Kloeden \& A. J. McLean (Eds.), Alcohol, drugs and traffic safety $T^{\prime} 95$ (pp. 213-221). Adelaide, Australia: Road Accident Research Unit.

Shaunak, S., O'Sullivan, E., \& Kennard, C. (1995). Eye movements. Neurological Investigations, Neurosurgery, \& Psychiatry, 59, 115-125.

Wayfinder-VR [On-line] (1995). Retrieved 2/95 at: www.pcweb.com/ $\mathrm{pni} / \mathrm{oem} / \mathrm{way}_{\mathrm{N}} \mathrm{vr} /$ data.htm

YAMADA, M. (1994). Head and eye coordination analysis and a new gaze analyser developed for this purpose. In G. d'Ydewalle \& J. van Rensbergen (Eds.), Visual and oculomotor functions (pp. 423-434). Amsterdam: Elsevier.

YounG, L. R., \& SHEENA, D. (1975). Survey of eye movement recording methods. Behavior Research Methods \& Instrumentation, 7, 397429.

\section{NOTES}

1. The apparatus was developed at the Institute of Psychology III, University of Würzburg, Germany.

2. Since the voltage data for the left and right eye are highly correlated, a sum score was used as a predictor.

3 . Since the data of the present experiment (the voltage data and the eye position for any given sampling time) are not independent random variables, the equations are only approximately correct. Especially the term $\left(y_{j}-m_{x}\right)^{2} / N s_{x}^{2}$ is incorrect, since the sample size $N$ is linearly dependent on the sampling frequency. A more conservative estimation might be to consider each degree of gaze angle as a separate independent variable $(N=13)$. In this worst case the confidence interval for a $6^{\circ}$ gaze angle would result in $C I=y_{j} \pm 1.87$.

(Manuscript received November 10, 1998 revision accepted for publication August 10, 1999.) 